\title{
In Vitro wound healing model: effects of chitosan films loaded with gentamicin and silver sulfadiazine on the wound filling rate
}

\begin{abstract}
Currently, animals use in research experiments has been widely questioned by Animal Protect Institutions and Organizations. Moreover, preliminary tests and scientific validation of research hypothesis are required before in vivo tests approval. Thus, animal testing could take a long time. For all these reasons, the development of an in vitro test that could simulate and/or replace animal research would be very interesting. The aim of this work was the development of an in vitro wound healing model for evaluation of the healing potential of drug-loaded cross-linked chitosan films. MG63 , a cell line derived from human osteosarcoma, was used as cell line model. The monolayer was cultured and when $100 \%$ confluence was reached, a wound was created using a scraper. The healing process was evaluated for 21 days and cells were stained after $0,7,14$ and 21 days of incubation. Wounds were treated with chitosan film $(\mathrm{CH})$, chitosan-bisulfite blocked diisocyanate cross-linked film $(\mathrm{CH}-\mathrm{X})$, chitosan cross-linked film loaded with gentamicin (CH-X-GE) and chitosan cross-linked film loaded with silver sulfadiazine (CH-X-SS). The effects of chitosan $(\mathrm{CH})$, bisulfite blocked diisocyanate (BBDI), gentamincin (GE) and silver sulfadiazine (SS) on the wound healing response were microscopically analyzed by the wound filling rate. $\mathrm{CH}$ and $\mathrm{CH}-\mathrm{X}$ films did not show deleterious effects on cell growth compared to the positive control (no film) during the studied period. $\mathrm{CH}-\mathrm{X}$-GE demonstrated a reduced wound filling rate at the first week due to the burst release of gentamicin at this period. $\mathrm{CH}-\mathrm{X}$-SS showed reduced wound filling rates during all period studied probably due to silver cytotoxicity and its accumulation by the cells. Finally, in vitro wound healing rates were compared to in vivo results reported in the Literature. Similar behavior was observed, suggesting that the proposed in vitro wound healing model could potentially replace preliminary in vivo studies.
\end{abstract}

Keywords: in vitro model, wound healing, wound dressing, chitosan, gentamicin, silver sulfadiazine.
Volume 2 Issue 3 - 2017

\author{
Maria Gabriela Nogueira Campos, ${ }^{1,3}$ Henry \\ Ralph Rawls, ${ }^{2}$ Lucia Helena Innocentini Mei, ${ }^{3}$ \\ Neera Satsangi ${ }^{4}$ \\ 'Federal University of Alfenas, Brazil \\ ${ }^{2}$ Comprehensive Dentistry, University of Texas Health Science \\ Center at San Antonio, USA \\ ${ }^{3}$ Department of Materials Engineering and Bioprocess, State \\ University of Campinas, Brazil \\ ${ }^{4}$ Innovative Research Solutions, Inc., USA
}

Correspondence: Maria Gabriela Nogueira Campos, Federal University of Alfenas, Institute of Science and Technology, Rodovia José Aurélio Vilela, I 1999, Poços de Caldas/MG, Brazil, Tel +55-35-3697-4600, Fax + 55-35-3697-4602, Email nogueiracamp@gmail.com

Received: April 27, 2017| Published: May 16, 2017
Abbreviations: $\mathrm{CH}$, chitosan film; $\mathrm{CH}-\mathrm{X}$, chitosan cross-linked film; BBDI, bisulfite blocked diisocyanate; GE, gentamicin; SS, silver sulfadiazine; $\mathrm{CH}-\mathrm{X}-\mathrm{GE}$, chitosan cross-linked film loaded with gentamicin; $\mathrm{CH}-\mathrm{X}-\mathrm{SS}$, chitosan cross-linked film loaded with silver sulfadiazine

\section{Introduction}

Animal testing or animal research refers to the use of animals in research experiments. Worldwide, about 100 million animals are used annually and either killed during the experiments or subsequently euthanized. ${ }^{1}$ Animal research is mainly carried out inside universities, medical schools and pharmaceutical companies, although some commercial facilities provide animal-testing services to industry. ${ }^{2}$ Groups that supports animal research affirm that it has played a vital role in every major medical advance and that many major developments, such as penicillin (mice), organ transplant (dogs), and poliomyelitis vaccine (mice, monkeys) involved animal researches. ${ }^{3}$ However, the topic is controversial. Opponent groups argue that animal testing is unnecessary, poor scientific practice, poorly regulated, that the costs outweigh the benefits, or that animals have an intrinsic right not to be used for experimentation. ${ }^{4}$ Nevertheless, most scientists and governments agree that animal testing should cause as little suffering to animals as possible, and that animal tests should only be performed where necessary, respecting the $3 \mathrm{R}$ 's principle: Reducing, Replacement and Refinement of the use of animals in research. ${ }^{5}$ In this context, the development of an in vitro test that could replace preliminary animals testing or serve as pre-screening before in vivo experiments is an interesting, current and crucial research field.

Wound healing is a complex process that involves five phases (hemostasis, inflammation, cellular migration and proliferation, protein synthesis and wound contraction, and remodeling), but only three major phases are effectively considered due to overlap of phases. ${ }^{6}$ Inflammatory, proliferative, and remodeling phases are associated with considerable complexity that involves soluble mediators, extracellular matrix formation, and parenchymal cell migration. ${ }^{7}$ Nevertheless, the primary goal of wound healing is timely wound closure, which is directly related to the wound filling rate. ${ }^{6,8}$ Additional help might be provide for wound healing in order to accelerate wound closure and/ or to improve the regenerated tissue quality. Several approaches on wound healing treatment are currently available, such as grafts (auto, allo and xenografts), use of donor keratinocytes, cultured epithelial autografts, delivery of growth factors and other molecules, use of stem cells and wound dressings. ${ }^{6,7}$ Wound dressings are most commonly used in wounds treatment due to the limitations of grafts (donor site, 
surgical complications, rejection, graft contraction and stability of the graft) and autologous cell culture (the time required to culture and prepare sheets of cells for grafting limit its use). ${ }^{6}$ In addition to its general functions, such as covering and protecting the wound against infection, avoiding wound dehydration and allowing woundenvironment gas/fluid exchanges, an ideal wound dressing should also serve as a matrix for cell adhesion and proliferation, as well as a vehicle to delivery growth factors and antibiotics. ${ }^{6-9}$ There are a number of wound dressing based on polymeric materials available on market, and chitosan is the most used polymer for this application. ${ }^{6}$

Chitosan is a biopolymer derived from chitin, the second most abundant polysaccharides found in nature. ${ }^{10}$ Due to its interesting biological properties such as biocompatibility, biodegradability, antibacterial activity and bio-adhesion, it has been widely studied and applied as a biomaterial in the biomedical area. ${ }^{10,11}$ Biomedical applications of chitosan involve implants, scaffolds for tissue engineering, skin substitutes and drug delivery vehicles. ${ }^{12-14}$ To increase the timeframe and consistency of kinetics of drug delivery from chitosan vehicles, crosslinking might be necessary. ${ }^{15}$ Several chemical agents are available for the crosslinking of chitosan: glutaraldehyde, genipin, epichlorohydrin, sulfuric acid, etc.; but glutaraldehyde is the molecule most commonly used as chitosan cross-linker. On the other hand, there are concerns over the toxicity of residual glutaraldehyde, which may compromise the biocompatibility of chitosan delivery system. ${ }^{15}$ A water-soluble bisulfite blocked diisocyanate has been recently prepared and used as crosslinking agent for chitosan. ${ }^{16}$ This bisulfite blocked diisocyanate is devoid of any toxic effects of the relative diisocyanate and preferably reacts with amine groups of chitosan by urea linkage. ${ }^{15,16}$

Therefore, in this present work, we evaluated the healing potential of chitosan and chitosan-blocked hexamethylene diisocyanate crosslinked films loaded with gentamicin and silver sulfadiazine on an in vitro wound healing model. For the in vitro model, human MG-63 cells, a cell line derived from an osteosarcoma, were seeded in sixwell tissue culture plates (Corning Co, USA). The seeded cells were incubated in $\alpha$-MEM (Minimum Essential Media Alpha Medium) with $5 \%$ fetal bovine serum (FBS) and humidity atmosphere of 5\% $\mathrm{CO} 2$ and $95 \%$ air at $37^{\circ} \mathrm{C}$. After cells reached confluence, wounds were created across the surface of each well (Figure 1A) and the media were replaced. The wounds were then microscopically examined to ensure that cellular and extra-cellular materials were completely removed from the wound sites (Figure 1B). At this moment, the chitosan-based films, previously prepared by solvent evaporation technique and sterilized using UV light (Table 1), were placed in each of the wells, and the positive control was a well with no film. The experiment was carried out on duplicates. The culture medium was replaced at every two days in order to provide essentials nutrients for cellular growth. The wound filling rate was observed for three weeks (21 days). After $0,7,14$ and 21days of incubation, films were removed from the wells and cells were stained with $20 \mathrm{ml}$ of T-blue stain for 2 minutes at $37^{\circ} \mathrm{C}$. After staining, all media were removed and cells were washed with $1 \mathrm{ml}$ of $0.1 \mathrm{M}$ phosphate buffer solution (PBS) $\mathrm{pH}$ 7.4. Then, PBS was removed and $1 \mathrm{ml}$ of formalin was added. Cells were kept at $4^{\circ} \mathrm{C}$ for 24 hours and, after this procedure, formalin was replaced for ethanol $70 \%$. At this moment, the wound filling rate was analyzed using a microscope with photography apparatus. The wound filling rate was qualitatively determined by the amount of cells that grown in each wound site at each analyzed period (Figure 2). According to Figure 2, $\mathrm{CH}$ and $\mathrm{CH}-\mathrm{X}$ films did not show deleterious effects on cell growth during the studied period if compared to the positive control (no film), suggesting that both films are biocompatible and BBDI are non-toxic to MG-63 cells. In fact, no significant difference was observed for the wound filling rate of these films, but stimulation on cells growth by $\mathrm{CH}$ at day 7 might be suggested. Ueno et al. ${ }^{17}$ reported that chitosan stimulated migration and proliferation of fibroblast and collagen production in a dog wound healing model. Moreover, wound healing experiments using mouse as model have shown that the application of chitosan hydrogel onto an open wound induced significant wound contraction and accelerated wound healing. ${ }^{18} \mathrm{Xu}$ et al. ${ }^{19}$ reported the biocompatibility of a genipin-chitosan hydrogel on a rabbit model. In addition, glycerol phosphate-chitosan hydrogel was assessed in experimental osteochondral joint defects in horses and did not cause relevant clinical effects, inflammatory response or toxic effects in the joints. ${ }^{20}$ Chitosan films were also evaluated on wound repair of horse distal limb and they had not interfered on healing time in the equine model ${ }^{21}$ suggesting compatible results of in vivo wound model and this reported in vitro wound model. On the other hand, the gentamicinloaded crosslinked chitosan film CH-X-GE showed inferior wound filling rate when compared to positive control in the first week. Lin et al. ${ }^{22}$ reported that application of $2 \%$ gentamicin eye drops in porcine cornea significantly disturbed the corneal epithelial healing rate. Cooper et al. ${ }^{23}$ investigated the cytotoxic effects of gentamincin on human fibroblasts and keratinocytes, which play an important role in wound healing, and found profound effects of gentamicin on these cells. Bertolaso et al. ${ }^{24}$ reported the biochemical mechanism underlying gentamicin cytotoxicity in OC-k3 cells. Although gentamicin ototoxicity and neuphrotoxicity have been extensively studied, the dose-dependent deleterious effects of gentamicin have not been well established yet. ${ }^{25,26}$ Hancock et al. ${ }^{27}$ studied the retinal toxicity of gentamicin in vivo and in vitro and reported reversible toxic effects in short-term exposure to gentamicin, while irreversible retinal damages were observed after prolonged gentamicin treatment, suggesting besides dose-dependent, toxicity of gentamicin is also time-dependent. After 14 and 21 days of incubation, CH-X-GE film deleterious effects on wound filling were not observed anymore in our in vitro wound healing model ( $\mathrm{CH}-\mathrm{X}-\mathrm{GE}$ wound filling rate is similar to positive control, $\mathrm{CH}$ and $\mathrm{CH}-\mathrm{X}$ films). This can be attributed to the decrease on gentamicin concentration in the media, since burst release happened in the first week and then minimal amount of gentamicin was controlled released by $\mathrm{CH}-\mathrm{X}-\mathrm{GE}$ films during the following two weeks. ${ }^{28}$ Again, the proposed in vitro wound healing model showed analogous results to those found in vivo, suggesting a dose and time-dependent effect of gentamicin on the wound filling rate. Contrary, silver sulfadiazine-loaded crosslinked chitosan film $\mathrm{CH}-\mathrm{X}-\mathrm{SS}$ showed reduced wound filling rate during all the analyzed period. Silver cytotoxicity id related to its accumulation in the body. ${ }^{29}$ Thus, the decrease on the amount released by the CH-X-SS trough the weeks does not reduce the cytotoxicity observed at 7,14 and 21 days of incubation. Lee et al. ${ }^{30}$ also reported the cytotoxic effect of silver sulfadiazine on $\mathrm{HaCaT}$ cells and the delayed epithelialization in an animal model. In addition, $\mathrm{Mi}$ et al..$^{31}$ observed a marked inhibition of 3T3 fibroblasts growth caused by $1 \%$ silver sulfadiazine cream treatment. According to authors, fibroblasts inhibition was significantly reduced by the use of silver sulfadiazine incorporated to an asymmetric chitosan membrane. ${ }^{31}$ Poon et al. ${ }^{32}$ studied the cytotoxic effects of silver on keratinocytes and fibroblasts in vitro. According to their results, silver is highly toxic to both keratinocytes and fibroblasts, which play an important role in wound healing process. Moreover, the cytotoxic dosage for skin cells was found to 
be similar to that for bacteria, suggesting the use of silver in wound treatments should be carefully evaluated since it could delay wound healing instead of accelerate it. ${ }^{32}$ Several in vivo studies using human and animal burn wounds report the side effects of silver sulfadiazine topical treatment, ${ }^{33-35}$ which corroborate with the results found in our in vitro wound healing model.

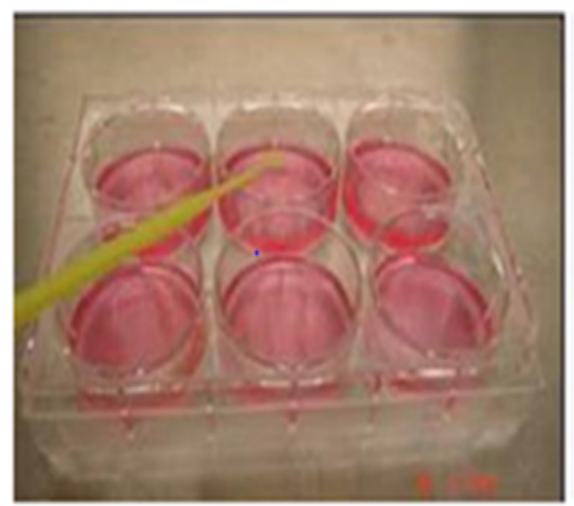

A. Cells being removed by a scraper in order to create a wound

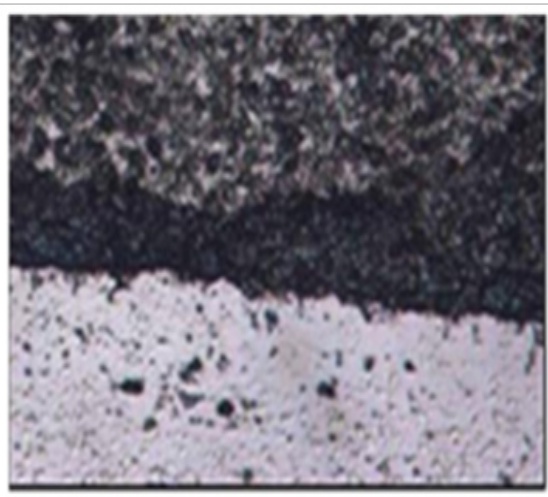

B. In vitro wound created after cell removal using a scraper (day 0). Cells are stained, while the wound site is not.

Figure I In vitro wound healing model apparatus.

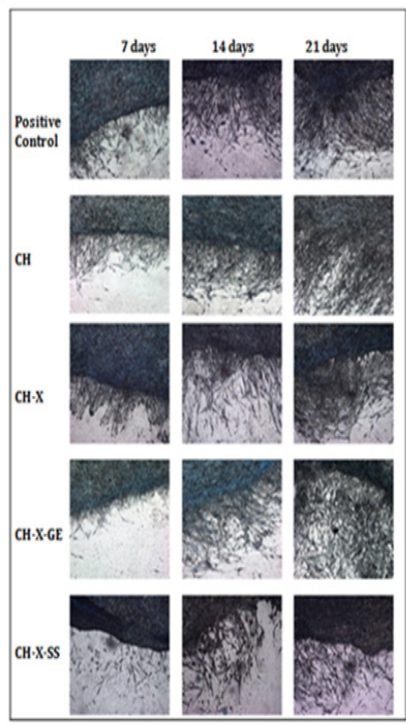

Figure 2 Wound healing results after at 7, 14 and 21 days of incubation.
Table I Preparation of chitosan-based films for in vitro wound healing response evaluation.

\begin{tabular}{ll}
\hline Sample & Film preparation/composition \\
\hline & In a $250 \mathrm{~mL}$ flask, I.5g of high molecular weight chitosan, \\
& $>75 \%$ deacetylated (Sigma-Aldrich, USA) was dissolved in \\
& $100 \mathrm{ml}$ of $\mathrm{I} .0 \%$ acetic acid solution.After total dissolution, \\
& the resulted solution was cast in the molds and the \\
& films were obtained by solvent evaporation at room \\
& temperature.
\end{tabular}

In a $250 \mathrm{~mL}$ flask, $0.8 \mathrm{~g}$ of blocked bisulfite diisocyanate ${ }^{15}$ was added to $100 \mathrm{ml}$ of $1.5 \%$ chitosan solution and heated up to $40^{\circ} \mathrm{C}$ under stirring for $24 \mathrm{~h}$. The cross-linked solution was cast in the molds and the films were obtained by solvent evaporation at room temperature.

In a $250 \mathrm{~mL}$ flask, $0.8 \mathrm{~g}$ of blocked bisulfite diisocyanate $\mathrm{e}^{15}$ was added to $100 \mathrm{ml}$ of $1.5 \%$ chitosan solution and heated up to $40^{\circ} \mathrm{C}$ under stirring for crosslinking. After $24 \mathrm{~h}, 1.0 \%$

$\mathrm{CH}-\mathrm{X}-\mathrm{GE}$ gentamicin sulfate $(\mathrm{w} / \mathrm{v})$ was added and the solution was homogenized under stirring for another $24 \mathrm{~h}$. Then, the solution was cast in the molds and the films were obtained by solvent evaporation at room temperature.

In a $250 \mathrm{~mL}$ flask, $0.8 \mathrm{~g}$ of blocked bisulfite diisocyanate ${ }^{15}$ was added to $100 \mathrm{ml}$ of $1.5 \%$ chitosan solution and heated up to $40^{\circ} \mathrm{C}$ under stirring for crosslinking. After $24 \mathrm{~h}, 5.0 \%$

$\mathrm{CH}-\mathrm{X}-\mathrm{SS}$ silver sulfadiazine $(\mathrm{w} / \mathrm{v})$ were added and the solution was homogenized under stirring for another $24 \mathrm{~h}$. Then, the solution was cast in the molds and the films were obtained by solvent evaporation at room temperature.

*All films were sterilized by exposure to UV light for 2 hours each side.

$\mathrm{CH}$, chitosan film; $\mathrm{CH}-\mathrm{X}$, chitosan cross-linked film; $\mathrm{CH}-\mathrm{X}-\mathrm{GE}$, chitosan crosslinked film loaded with gentamicin; $\mathrm{CH}-\mathrm{X}-\mathrm{SS}$, chitosan cross-linked film loaded with silver sulfadiazine.

\section{Conclusion}

In vitro wound healing model results have shown to be consistent to those obtained in animal studies reported in the Literature. Therefore, it could potentially replace preliminary animal testing and/or serve as pre-screening before in vivo experiments, collaborating to the $3 \mathrm{R}$ 's principle of reduction, replacement and refinement of animal tests.

\section{Acknowledgements}

The authors thank CAPES and CNPq for financial support.

\section{Conflict of interest}

The author declares no conflict of interest.

\section{References}

1. The Ethics of research involving animals, Nuffield Council on Bioethics, UK: Springer; 2005. 45 p.

2. National Research Council (US) and Institute of Medicine (US) Committee on the Use of Laboratory Animals in Biomedical and Behavioral Research Use of laboratory animals in biomedical and behavioral research, USA: The National Academies Press; 1998

3. Animal Research. Foundation for Biomedical Research, USA: Springer; 2017. 
4. Festing S, Wilkinson R. The ethics of animal research. Talking point on the use of animals in scientific research. EMBO Rep. 2007;8(6):526530 .

5. Review of cost benefit assessment in the use of animals in research., UK Animal Procedures Committee; 2003.

6. Dreifke MB, Jayasuriya AA, Jayasuriya AC. Current wound healing procedures and potential care. Mater Sci Eng C Mater Biol Appl. 2015;48(1):651-662.

7. Morton LM, Phillips TJ. Wound healing and treating wounds: Differential diagnosis and evaluation of chronic wounds. J Am Acad Dermatol. 2016;74(4):589-605.

8. Daniel Harper, Alistair Young, Clare Ellen McNaught. The physiology of wound healing. Surgery. 2014;32(9):445-450.

9. Korting HC, Schöllmann C, White RJ. Management of minor acute cutaneous wounds: importance of wound healing in a moist environment. J Eur Acad Dermatol Venereol. 2010;25(2):130-137.

10. Keisuke Kurita. Chemistry and application of chitin and chitosan. Polym Degrad Stab. 1998;59:117-120.

11. Berger J, Reist M, Mayer JM, et al. Structure and interactions in covalently and ionically crosslinked chitosan hydrogels for biomedical applications. Eur J Pharm Biopharm. 2004;57(1):19-34.

12. Hoemann CD, Sun J, Légaré A, et al. Tissue engineering of cartilage using an injectable and adhesive chitosan-based cell-delivery vehicle Osteoarthritis Cartilage. 2005;13(4):318-329.

13. Denkbaş EB, Oztürk E, Ozdemir N, et al. Norfloxacin-loaded chitosan sponges as wound dressing material. J Biomater Appl. 2004;18(4):291303

14. Ueno H, Mori T, Fujinaga T. Topical formulations and wound healing applications of chitosan. Adv Drug Deliv Rev. 2001;52(2):105-115.

15. Campos MGN, Satsangi N, Rawls HR, et al. Chitosan cross-linked films for drug delivery application. Macromol Symp. 2009;279(1):169-174.

16. Sheng Lin Gibson, Howard J Walls, Scott B Kennedy, et al. Reaction kinetics and gel properties of blocked diisocyanate crosslinked chitosan hydrogels. Carbohydr Polym. 2003;54(2003):193-199.

17. Ueno H, Yamada H, Tanaka I, et al. Accelerating effects of chitosan for healing at early phase of experimental open wounds in dogs. Biomaterials. 1999;20(15):1407-1414.

18. Ishihara M, Nakanishi K, Ono K, et al. Photo crosslinkable chitosan as a dressing for wound occlusion and accelerator in healing process. Biomaterials. 2002;23(3):833-840.

19. Xu J, Strandman S, Zhu JX, Barralet J, et al. Genipin-crosslinked catechol-chitosan mucoadhesive hydrogels for buccal drug delivery. Biomaterials. 2015;37:395-404.

20. Martins EAN, Baccarin RYA, Moraes APL, et al. Evaluation of chitosan-glycerol phosphate in experimental osteochondral joint defects in horses. J Mol Genet Med. 2015;S4:002.
21. Martins EAN, Invernizzi MS, Campos MGN, et al. Chitosan film in horses with experimentally induced skin wound. Ciencia Rural. 2013;43(10):1824-1830.

22. Lin CP, Boehnke M. Effect of fortified antibiotic solutions on corneal epithelial wound healing. Cornea. 2000;19(2):204-206.

23. Cooper ML, Laxer JA, Hansbrough JF. The cytotoxic effects of commonly used topical antimicrobial agents on human fibroblasts and keratinocytes. J Trauma. 1991;31(6):775-782.

24. Bertolaso L, Bindini D, Previati M, et al. Gentamicin-induced cytotoxicity involves protein kinase $\mathrm{C}$ activation, glutathione extrusion and malondialdehyde production in an immortalized cell line from the organ of corti. Audiol Neurootol. 2003;8(1):38-48.

25. Marais J, Rutka JA. Ototoxicity and topical ear drops. Clin Otolaryngol. 1998;23(4):360-367.

26. Brummett RE, Fox KE. Aminoglycoside-induced hearing loss in humans. Antimicrob Agents Chemother. 1989;33(6):797-800.

27. Hancock HA, Guidry C, Read RW, et al. Acute aminoglycoside retinal toxicity In Vivo and In Vitro. Invest Ophthalmol Vis Sci. 2005;46(12):4804 4808 .

28. Campos MG, Rawls HR, Innocentini Mei LH, et al. In vitro gentamicin sustained and controlled release from chitosan cross-linked films. $J \mathrm{Ma}$ ter Sci Mater Med. 2009;20(2):537-542.

29. Shanmugasundaram N, Sundaraseelan J, Uma S, et al. Design and delivery of silver sulfadiazine from alginate microspheres-impregnated collagen scaffold. J Biomed Mater Res B Appl Biomater. 2005;77(2):378-388

30. Cho Lee AR, Leem H, Lee J, et al. Reversal of silver sulfadiazine-impaired wound healing by epidermal growth factor. Biomaterials. $2005 ; 26(22): 4670-4676$

31. Fwu Long Mi, Yu bey Wu, Shin Shin Shyu, et al. Asymmetric chitosan membranes prepared by dry/wet phase separation: a new type of wound dressing for controlled antibacterial release. J Memb Sci. 2003;212(12):237-254.

32. Poon VK, Burd A. In vitro cytotoxicity of silver: implication for clinical wound care. Burns. 2004;30(2):140-147.

33. Fuller FW. The side effects of silver sulfadiazine. J Burn Care Res. 2008;30(3):464-470.

34. Coombs CJ, Wan AT, Masterton JP, et al. Do burn patients have a silver lining? Burns. 1992;18(3):179-184.

35. Wan AT, Conyers RA, Coombs CJ, et al. Determination of silver in blood, urine, and tissues of volunteers and burn patients. Clin Chem. $1991 ; 37(10$ Pt 1):1683-1687. 